\title{
The effect of animal assisted therapy on participants with dementia in a community respite program
}

\author{
William Stuart Pope, Morgan Yordy, Chih-hsuan Wang \\ School of Nursing, Auburn University, United States
}

Received: November 14, 2018

Accepted: January 13, 2019

Online Published: January 18, 2019

DOI: $10.5430 /$ jnep.v9n5p58

URL: https://doi.org/10.5430/jnep.v9n5p58

\begin{abstract}
Cognitively impaired older adults pose challenges that include communication barriers that may contribute to social isolation of the individual and frustration by both the individual and caregivers. Healthcare professionals must to be prepared to speak to the distinctive requirements of this population. Innovative strategies are needed to improve the ability of caregivers and health professionals to establish effective communication. Animal Assisted Therapy (AAT) is a complementary therapy that shows promise in providing emotional and social benefits to older adults in both clinical and community settings. This project aims to describe the benefits of incorporating AAT within a community respite program to enhance social engagement of cognitively impaired adults. In this project a group of subjects were exposed to two situations in an unsystematic order, visits with a dog and visits without a dog. The purpose was to compare each visit and its effect in improving engagement in those attending a community respite program. Throughout the study, respite attendees were encouraged to engage with dogs or the human visitor. In this study, AAT enhanced social engagement.
\end{abstract}

Key Words: Animal assisted therapy, Respite, Community

\section{INTRODUCTION}

The number of older adults is rising. This rise has an associated increase in those with dementia. Dementia and all types of intellectual deficiency have benn shown to produce a negative outcome on the value of living. Dementia develops slowly and is depicted by a decrease in the cognitive, executive and function of memory while remaining in a state of full alertness. Alzheimer's disease (AD) is the predominant type of cognitive impairment. AD is an incurable, progressive disease and because there are no preventative measures or cure, interventions must focus on improving function and quality of life. ${ }^{[1]}$ Dementia or cognitive impairments challenge communities, caregivers, and medical personnel to individualize health education and health promotion for the older adults to meet their complex health needs. ${ }^{[2-4]}$ Currently more than
5 million people live with Alzheimer's or other dementias and by 2050 , this number could increase to 14 million. ${ }^{[5]}$ With the population aging and many afflicted with cognitive impairments, family, friends, healthcare providers, and the community are in a position to care for the elderly and assist with activities of daily living that are cost effective and meaningful to the individual. ${ }^{[2,4]}$ Cognitively impaired older adults pose challenges of social isolation of the individual and frustration by both the individual and caregivers. Healthcare professionals must to be prepared to address the unique needs of this population. Innovative strategies are needed to promote social engagement among this vulnerable population and improve the ability of caregivers and health professionals to establish effective communication. Animal-assisted therapy (AAT) shows promise in providing

\footnotetext{
*Correspondence: William Stuart Pope; Email: wsp0002@auburn.edu; Address: School of Nursing, Auburn University, United States.
} 
emotional and social benefits to older adults in both clinical and community settings. This project aims to describe the benefits of incorporating AAT within a community respite program to enhance social engagement of cognitively impaired adults.

\subsection{Meaningful activities}

Dementia is a costly disease in the United States with estimates of around $\$ 50,000$ annually per case relating to dementia care. ${ }^{[6]}$ Over 16 million caregivers provide voluntary care to those living with Alzheimer's or other dementias totaling over $\$ 230$ billion in 2017. ${ }^{[5]}$ Due to high associated cost to care for those presenting with this disease, cost effective activities are imperative to implement into dementia care programs.

Providing meaningful activities for older adults, especially those with cognitive impairments, is paramount for healthy aging. Studies show engaged participation leads to less boredom, depression, and loneliness. ${ }^{[7]}$ Also, engagement in activities has shown to improve psychological and physical well-being and slow the progression of the disease. ${ }^{[8]}$ More community programs are needed to provide activities to enhance communication, social behavior engagement, and improve quality of life. ${ }^{[9]}$ Support services exist and respite in an adult day program provides safe environments with improved patient outcomes. ${ }^{[10]}$

The diagnosis of cognitive impairment is life altering. Even in early stage dementia, physical and psychological changes become problematic for self-care and quality of life. ${ }^{[3]}$ Family, friends, and healthcare providers often seek activities to enhance life satisfaction, yet studies suggest underutilization of programs. A survey of 113 caregivers confirmed the burden and stress involved in a client with cognitive impairment living at home, yet many caregivers believed using respite programs would result in negative outcomes or no positive outcomes. $^{[4]}$

Highlighting the benefits to community respite programs which incorporate activities to stimulate those inflicted with early cognitive impairment could increase utilization. ${ }^{[4]} \mathrm{Al}-$ though not every activity will appeal to every older person nor should the older person be engaged in a stimulating activity all the time, there is a need to facilitate participation in meaningful activities. ${ }^{[11]}$ Persons living with dementia often lack opportunities especially with daytime activities and social companionship. ${ }^{[12]}$ Han et al. completed a synthesis of 34 qualitative studies. One common theme discovered was the desire to continue engagement and activities that support habits and leisure activities to promote talking of past experiences. ${ }^{[12]}$ A survey of 216 caregivers found that perPublished by Sciedu Press sons with dementia enjoyed the same activities as the general older population. ${ }^{[13]}$ Findings from these studies suggest a need to support activity that are diverse and pleasurable, ${ }^{[8,12]}$ tailor activities to the level of function to build on personal abilities, ${ }^{[14]}$ and focus on cost- benefit of the programing. ${ }^{[15]}$

\subsection{Animal assisted therapy}

Animal assisted therapy shows promise as a means to enhance social engagement for the cognitively impaired older adult. AAT is "a goal oriented, planned, and structured therapeutic intervention directed and/or delivered by health, education, or human service professionals" (p. 416). ${ }^{[16]}$ Animals are found to assist in relationship building and calm people. ${ }^{[17]}$ Older adults that own a pet report less loneliness and have increased activity. Unfortunately, many living with dementia are unable to care for a pet. Pets can be costly to those already on a fixed income and as the disease progresses, many will not be able to care for a pet, therefore, interventions and therapy that incorporates animals has grown in popularity. ${ }^{[19]}$ AAT has been shown to enhance communication between patient and caregiver and provide comfort and support for patients. ${ }^{[20]}$ Animals stimulate sensory perception and provide emotional support without poly-pharmacological and high co-morbidity treatments. ${ }^{[21]}$

There is rising awareness in non-pharmacological interventions for those living with dementia that should be considered for all types and severities. AAT is a good form of treatment as the participant interacts with a trained animal and handler. This is seen in a crossover study conducted among 44 nursing home residents living with dementia where observations of animal and human engagement were documented. Engagement and behavior did establish affirmative alterations in AAT over visits from only a human. A decreased amount of distress was seen as well. A decrease in voiced aggression and a rise in the evidence of gratification was realized in AAT over human only visits. This posits that visits with an animal was effective in increasing social engagement with people with dementia. ${ }^{[22]}$ Swall, Ebbeskog, Hageline, \& Fagerberg studied nine dog-handlers trained in AAT who visited older persons with dementia. ${ }^{[23]}$ The dog-handlers reported AAT brought respite from illness by tailoring visits for participants that were person-centered to increase quality of life and activity in those living with dementia. A meta-analysis of ten studies of animal-assisted interventions patients with cognitive impairment showed a statistically significant decrease in the behavioral and psychological symptoms of dementia such as disturbed behavior, mood, agitation and depression. The results of this study suggests that animal assisted therapy is a suitable treatment for those with cognitive impairment. [24] A 12-week study with 58 participants found significant im- 
provement in the quality of life and depression with animal assisted interventions compared to the control group. The control group's symptoms slowly exacerbated during the study. ${ }^{[25]}$

Early and accessible community services to promote wellbeing are needed, ${ }^{[26]}$ yet dementia care is financially debilitating for patient and caregiver. ${ }^{[6,15]}$ Community stakeholders and caregivers seek activities to enhance life satisfaction, ${ }^{[13]}$ improve psychological and physical well-being, ${ }^{[8]}$ and can be utilized throughout all types and severities of dementia without being too costly. ${ }^{[27]}$ AAT is a beneficial activity that is not costly to the end user. Many trained dog-handler teams volunteer their time to visit community groups and participants without payment. ${ }^{[28]}$ AAT is a meaningful and cost effective activity to utilize in a community respite program.

\section{METHOD}

In this project a group of subjects were exposed to two situations in an unsystematic order, visits with a dog and visits without a dog. The purpose was to compare each visit and its effect in improving engagement in those attending a community respite program. Throughout the study AAT, members were encouraged to engage with dogs or the human visitor.

\subsection{Ethical considerations}

Institutional Review Board (IRB) and Institutional Animal Care and Use Committee (IACUC) approval were obtained for the project. Approval was also acquired from the contributing community respite program. Participants' relatives were communicated with by investigators to conclude if they were interested in participation in the program. After agreement, an in person conference was made by investigators with each family to describe the program and discuss issues. Consent was then obtained from relatives. Explanation was given to the participants about the program and those that were qualified gave spoken acceptance. Members also agreed to participation at each therapy session or it was not conducted.

\subsection{Participants}

Fourteen participants in a community respite program with a diagnosis of cognitive impairment; 8 males and 6 females, from 65 to 86 years old, were observed during human interaction visits and AAT visits. Confirmation of their diagnosis was completed through interviews with participants families. The level of dementia along the spectrum from mild to severe, but all participants were required to tend to their own toileting.

\subsection{Study depiction}

Members were observed in random order for animal visits or human interface. Regardless of type of visit as listed above, observations were for 5 minutes during anallotted time during the 15-minute interaction visit. A discretely positioned, trained observer recorded the positive and negative social behaviors and engagement at 1 minute intervals over a 5minute time frame using the Social Behaviors Check. ${ }^{[29]}$ The overall level of engagement during the session will be noted by the observer using a Likert Scale ranging from low to high engagement.

\subsection{Animal intervention}

Three AAT trained dogs, ages one, eight and ten years old, were used to involve the participants. The program was completed using dogs between 40 and 70 pounds. Animals used in the AAT portion of this study were examined by the progam veterinarian to certify they were free from disease and that all immunizations were up-to-date. The dogs were bathed and groomed each day before participation. Throughout the study, an animal handler accompanied the canine during the period, but did not attempt engage the participant in conversation other than to encourage involvement with the dog. AAT took place in the social room. The dogs were permitted to involve the participants any way the participant wanted. The human-animal bond was used to guide the visits, based on the mental and physical capabilities ability of participants.

\subsection{Human visits}

The human interaction consisted of a student engaging the participant in verbal interaction. This offers equivalent personal attention and is practical. Where engagement proves impossible, the student remained with the participant and follow whatever they do for the required period. The student was a junior/senior leveling nursing student under the supervision of a faculty member.

\subsection{Instruments}

The Social Behaviors Check List was a direct observational tool with proven validity in persons demonstrating cognitive impairment. ${ }^{[29]}$ It was modified for a previous AAT study with elderly nursing home residents by AUSON faculty and recently published. ${ }^{[22]}$ This instrument contains 13 social behaviors (six positive and seven negative). These behaviors are witnessed and recorded in one-minute periods and a total social behavior score was calculated by measuring the variance between constructive and unconstructive behaviors noted during the canine visit. The social behaviors scores were statistically compared between the clients who interacted with AAT to those who just experienced student nurse 
interaction.

\subsection{Data collection}

After agreement and prior to appointments, the participant and/or relative completed a survey consisiting of gender, age range, and previous or past pet owndership to include pet type. For all visits, dog and human, an inconspicuously positioned observer used the Social Behaviors tool to record their surveillance during the intervention. To attain valid and reliable data the observers were given specific project training before the gathering of data.

\subsection{Data analysis}

The study compared the positive and negative behaviors of the participants when they were with human only or with dogs and human. Data was scored using descriptive statistics and paired samples $t$-test.

\section{Results}

Results indicated that participants' positive behaviors presented more when they were accompanied with dogs and human altogether, especially they laughed more $(t(12)=3.21$, $p=.01$, with large effect size Cohen's $d=0.88)$, they learned more $(t(12)=3.75, p=.003$, with large effect size Cohen's $\mathrm{d}=1.04)$, and they touching more $(t(12)=5.25, p<.001$, with large effect size Cohen's $d=1.46)$. As for the negative behaviors, participants did not present any spitting, biting, throwing, cursing, kicking, or grabbing during the observation. Participants' other negative behaviors presented no differences when they were or were not accompanied with dogs (see Table 1).

Table 1. Descriptive statistics and paired samples $t$-Test results

\begin{tabular}{|c|c|c|c|c|c|c|c|}
\hline \multirow{2}{*}{ Behavior } & \multicolumn{2}{|l|}{ Dog } & \multicolumn{2}{|c|}{ Human } & \multirow{2}{*}{$\begin{array}{l}t \\
(d f=12)\end{array}$} & \multirow{2}{*}{$p$} & \multirow{2}{*}{$\begin{array}{l}\text { Cohen's } d \\
\text { Effect Size }\end{array}$} \\
\hline & $M$ & $S D$ & $M$ & $S D$ & & & \\
\hline \multicolumn{8}{|l|}{ Positive } \\
\hline Smiling & 0.68 & 0.33 & 0.55 & 0.31 & 2.11 & .06 & 0.58 \\
\hline Laughing & 0.38 & 0.31 & 0.24 & 0.19 & 3.21 & $.01 * *$ & 0.88 \\
\hline Looking & 0.69 & 0.21 & 0.67 & 0.19 & 0.28 & .78 & 0.08 \\
\hline Leaning & 0.46 & 0.29 & 0.14 & 0.16 & 3.75 & $.003^{* *}$ & 1.04 \\
\hline Touching & 0.52 & 0.30 & 0.09 & 0.09 & 5.25 & $<.001 * * *$ & 1.46 \\
\hline Verbalizing/Crying & 0.62 & 0.27 & 0.66 & 0.26 & -0.47 & .65 & -0.13 \\
\hline Listening & 0.56 & 0.26 & 0.51 & 0.29 & 0.93 & .37 & 0.26 \\
\hline \multicolumn{8}{|l|}{ Negative } \\
\hline Screaming/Yelling & 0.002 & 0.01 & 0.00 & 0.00 & 1.00 & .34 & 0.28 \\
\hline Spitting & 0.00 & 0.00 & 0.00 & 0.00 & --- & --- & --- \\
\hline Biting & 0.00 & 0.00 & 0.00 & 0.00 & --- & --- & --- \\
\hline Throwing Something & 0.00 & 0.00 & 0.00 & 0.00 & --- & --- & --- \\
\hline Cursing & 0.00 & 0.00 & 0.00 & 0.00 & --- & --- & --- \\
\hline Kicking & 0.00 & 0.00 & 0.00 & 0.00 & --- & --- & --- \\
\hline Pushing/Swatting & 0.003 & 0.01 & 0.00 & 0.00 & 1.00 & .34 & 0.28 \\
\hline Making Strange or Negative Noises/Crying & 0.00 & 0.00 & 0.003 & 0.01 & -1.00 & .34 & -0.28 \\
\hline Grabbing & 0.00 & 0.00 & 0.00 & 0.00 & --- & --- & --- \\
\hline $\begin{array}{l}\text { Withdrawal/Purposeful Turning } \\
\text { Away/Verbalize Not Wanting to Interact }\end{array}$ & 0.03 & 0.04 & 0.02 & 0.04 & 0.55 & .59 & 0.15 \\
\hline
\end{tabular}

\section{Discussion}

Improving the quality of life for senior adults is a priority in many communities. AAT is an affordable means of improving the quality of life with this population. ${ }^{[28]}$ Although AAT and human interaction were successful in this study, AAT outperformed human interaction. But, as seen in other studies, both animal and human visits resulted in the increase in positive social behaviors. ${ }^{[20-23]}$

Respite programs are often underutilized. This underutilization is due in part to the family's belief that the program will result in negative outcomes. ${ }^{[4]}$ A control trial using stuffed dogs resulted in increased gratification and awareness in adults in a Alzheimer's daycare. Also noted in the trial 
were decreased sadness and anxiety. as well as a reduction in sadness and anxiety in Alzheimer's patients at an adult day center was observed. ${ }^{[29]}$ The interations seen with this study using AAT in a respite program were positive and participants were more engaged. In addition, the existing program also noted a significant rise in enjoyment. Negative behaviors were not present with either interaction; human or human with canine. Lastly, interventions that are cost effective are needed for this population. ${ }^{[15]}$ No cost was incurred by the respite program. The canine-handler teams volunteered their time to visit respite particpants.

\section{Conclusion}

With a growth in the elderly population there is an associated increasing population of those with dementia. This greatly affects the quality of life for these individuals as well as their families. AAT performed according to the national recommendations and following ethical principles is an effective, affordable, supportive, complimentary treatment to improve the engagement and value of life in people living with cognitive impairment.

\section{CONFLICTS OF INTEREST Disclosure}

The authors declare that there is no conflict of interest.

\section{REFERENCES}

[1] National plan to Address Alzheimer's Disease: 2015b Update. U.S. Department of Health and Human Services, National Institutes of Health. NIH. Available from: https://aspe.hhs.gov/national-plan-address-alzheim erT1textquoterights-disease-2015-update

[2] Older Adults. Health People 2020. Health People Web Site. Available from: https://www.healthypeople.gov/2020/topics-obj ectives/topic/older-adults

[3] Jao Y, Epps F, McDermott C, et al. Effects of support groups for individuals with early-stage dementia and mild cognitive impairment. Research in Gerontological Nursing. 2017; 10(1): 35-51. PMid:27665754 https://doi.org/10.3928/19404921-20160 726-01

[4] Phillipson L, Magee C, Jones SC. Why carers of people with dementia do not utilize out of home respite services. Health and Social Care in the Community. 2013; 21(4): 411-422. PMid:23496258 https://doi.org/10.1111/hsc. 12030

[5] Alzheimer's Association. Facts and Figures. 2018. Available from: https://www.alz.org/alzheimers-dementia/fact s-figures

[6] Hurd MD, Martorell P, Delavande A, et al. Monetary costs of dementia in the United States. The New England Journal of Medicine. 2013; 368: 1326-1334. PMid:23550670 https ://doi .org/10.1 056/NEJMsa1204629

[7] Cohen-Mansfield J, Dakheel-ali M, Marx M. Engagement in persons with dementia: the concept and its measurements. The American Journal of Geriatric Psychiatry. 2009; 17(4): 299-307.

[8] Roland KP, Chappell NL. Meaningful activity for persons with dementia: Family caregiver perspectives. American Journal of Alzheimer's Disease and Other Dementias. 2015; 30: 559-568. PMid:25788432 https://doi .org/10.1177/15333175155763 89

[9] Cai Y, Abrahamson K. Does exercise impact cognitive performance in community-dwelling older adults with mild cognitive impairment? A systematic review. Quality in Primary Care. 2015; 23(4): 214-222.

[10] Whitlatch CJ, Orsulic-Jeras S. Meeting the informational, educational, and psychosocial support needs of persons living with dementia and their family caregivers. The Gerontologist. 2018; 58(S1): S58-S73.

[11] Nyman SR. Meaningful activities for improving the wellbeing of people with dementia: Beyond mere pleasure to meeting fundamental psychological needs. Perspectives in Public Health. 2016; 136(2):
99-107. PMid:26933079 https : //doi .org/10.1177/17579139 15626193

[12] Han A, Radle J, McDowd JM, et al. Perspectives of people with dementia about meaningful activities: A synthesis. American Journal of Alzheimer's Disease and Other Dementias. 2016; 31(2): 115-123. PMid:26340962 https://doi.org/10.1177/15333175155988 57

[13] Menne HL, Johnson JD, Whitlatch CJ, et al. Activity preferences of persons with dementia. Activities, Adaptation, and Aging. 2012; 36: 195-213.

[14] Thwaite S, Henderson-Greene M. Towards meaningful activity and engagement. Journal of Dementia Care. 2013; 21(1): 18-19.

[15] Jutkowitz E, Gitlin LN, Pizzi LT. Evaluating willingness-to-pay thresholds for dementia caregiving interventions: Application to the tailored activity program. International Society for Pharmacoeconomics and Outcomes Research. 2010; 13(6): 720-725.

[16] Jegatheesan B, Beetz A, Ormerod E, et al. "The IAHAIO definitions for animal assisted intervention and guidelines for wellness of animals involved" in Handbook on Animal-Assisted Therapy, ed. A. H. Fine (New York, NY: Academic Press); 2015.

[17] Holttum S. Pets, animal-assisted therapy and social inclusion. Mental Health and Social Inclusion. 2018; 22(2): 65-71. https ://doi.or g/10.1108/MHSI-02-2018-0004

[18] Gee NR, Mueller MK, Curl AL. Human-animal interaction and older adults: An overview. Frontiers in Psychology. 2017; 8.

[19] Cherniack EP, Cherniack AR. The benefit of pets and animal-assisted therapy to the health of older individuals. Current Gerontology and Geriatric Research. 2014.

[20] Creagan E, Bauer B, Thomle B, et al. Animal-assisted therapy at Mayo Clinic: The time is now. Complementary Therapies in Clinical Practice. 2015; 21: 101-104. PMid:25900612 https ://doi .org/ $10.1016 / \mathrm{j}$.ctcp. 2015.03.002

[21] Bernabei V, De Ronchi D, La Ferla T, et al. Animal-assisted interventions for elderly patients affected by dementia or psychiatric disorders: A review. Journal of Psychiatric Research. 2013; 47: 762773. PMid:23369337 https://doi.org/10.1016/j.jpsychir es.2012.12.014

[22] Pope WS, Hunt C, Ellison K. Animal Assisted Therapy versus Human Interaction in the Improvement of Social Outcomes in Older Adults with Dementia. Journal of Nursing Education and Practice. 2016; 6(9). 
[23] Swall A, Ebbeskog B, Hagelin CL, et al. 'Bringing respite in the burden of illness'- Dog handlers' experience of visiting older persons with dementia together with a therapy dog. Journal of Clinical Nursing. 2016; 25: 2223-2231. PMid:27277921 https: //doi.org/10.1111/jocn. 13261

[24] Hu M, Zhang P, Leng M, et al. Animal-assisted intervention for individuals with cognitive impairment: A meta-analysis of randomized controlled trials and quasi-randomized controlled trials. Psychiatry Research. 2017; 260: 419-427.

[25] Olsen C, Pedersen I, Bergland A, et al. Effect of animal-assisted interventions on depression, agitation and quality of life in nursing home residents suffering from cognitive impairment or dementia: a cluster randomized controlled trial. Int J Geriatr Psychiatry. 2016; 31: 13121321. PMid:26807956 https://doi .org/10.1002/gps. 4436
[26] Nordgren L, Engstrom G. Effects of dog-assisted intervention on behavioural and psychological symptoms of dementia. Nursing Older People. 2014; 26(3): 31-38. PMid:24673326 https://doi.org/ 10.7748/nop2014.03.26.3.31.e517

[27] PetParners (n.d.) About. Pet Partners. Available from: https: //petpartners.org/

[28] Kongable LG, Buckwaltwer KC, Stolley J. the effects of pet therapy on the social behavior of institutionalized Alzheimer's clients. Archives of Psychiatric Nursing. 1989; 3: 191-198.

[29] Mossello E, Ridolfi A, Mello A, et al. Animal-assisted activity and emotional status of patients with Alzheimer's disease in day care. International Psychogeriatrics. 2011; 23(6): 899-905. PMid:21356158 https://doi.org/10.1017/S1041610211000226 